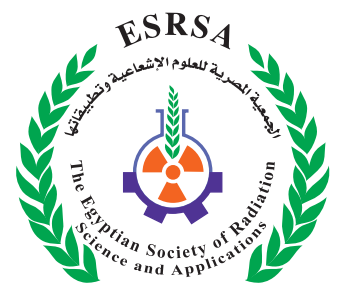

\title{
Radiation Synthesis of Methacrylic Acid/Poly (2-Ethyl-2-Oxazoline) Copolymer Hydrogel for Colon-Specific Drug Targeting Delivery System
}

\author{
Al-qudah, Y.H.F.
}

Received: 03/09/2018

Accepted: 17/03/2019

E.mail:Dr.yahyaqudah@bau.edu.jo

\section{ABSTRACT}

PH-sensitive hydrogels composed of Methacrylic acid and Poly (2-ethyl-2-oxazoline) (MAAc/PEOz) were prepared by means of $\gamma$-radiation induced graft copolymerization and crosslinking. The preparation parameters such as feed solution composition and concentration and irradiation dose were optimized. The effect of $\mathrm{pH}$ on the equilibrium of swelling for the prepared hydrogels was examined. Studying the diffusion's parameters and coefficient as a function of the $\mathrm{pH}$ values for the surrounding solution showed obvious changes in the swelling parameters as a result of changing the surrounding solution $\mathrm{pH}$ values. In order to estimate the ability of the prepared copolymer to be used as a colon-specific drug carrier, the release of indomethacin was monitored as a function of time at $\mathrm{pH} 3$ and $\mathrm{pH}$ 7. The observed results show the capability of the prepared hydrogel to protect the contained model drug at buffer solution of $\mathrm{pH} 1$ and start releasing the drug as soon as the hydrogel transferred

\section{KEYWORDS} to buffer solution of $\mathrm{pH}$ 7. Such data would recommend the prepared hy-

Copolymerization; drogel as a promising candidate for colon specific drug delivery system.

\section{Gamma-Radiation;}

Swelling Kinetics;

Drug Delivery System.

1. Al-Balqa' Applied University, Faculty of science, Department of Chemistry, Irbid, Jordan 


\section{INTRODUCTION}

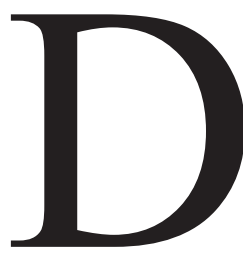

esigning and constructing of drug delivery systems (DDS) attract the attention of many researchers in the last decades (Streisand and Stanley 1995, Sakr, 1999; Sher et al., 2007; Sarpietro and Castelli, 2013; Szewczyk et al., 2019). The intelligent DDS have been developed to deliver drug effectively to the targeting site providing drug safety and efficiency (Davoodi et al., 2018, Fu, et al., 2018). Site specific drug delivery is important for macromolecular drugs such as proteins, which are very difficult to deliver through the conventional oral rotes because of their remarkable short half-life in vivo, their sensitivity to the acidic medium in the stomach, and in some cases undesirable pharmako kinetics (Aqil et al., 2013; Florence, 2018). One of the most important factors affecting the performance of the drug carrier in the release process is the water diffusion mechanism (Kohrs et al., 2018).

Hydrogels are water-swollen network of hydrophilic homopolymers or copolymers; it is interesting that they exhibit both liquid like properties and solid like properties. The liquid like properties result from the fact that its major constituent is water. However, the polymer exhibits solid like properties due to the network structure formed by crosslinking (Dragan, 2014; Ullah et al., 2015 and Mahinroosta et al., 2018). Polymers containing ionizable functional groups that respond to change in $\mathrm{pH}$ are called $\mathrm{pH}-$ sensitive polymers (Palmer et al., 2018). By generating the charge along the polymer backbone, the electrostatic repulsion results in an increase in the hydrodynamic volume of the polymer. The aforementioned characteristics of the polymeric gel make it a good candidate for being used as carrier for site specific drug release using the concept of $\mathrm{pH}$ as a trigger to release a drug in the colon based on the $\mathrm{pH}$ conditions that vary continuously down the GI tract (Wang et al., 2017 and Li et al., 2018). The release of the drug loaded in a polymeric gel takes place by migration of the drug to the surrounding medium by molecular diffusion through the microporous of the polymeric matrix. Fick's second law of diffusion is used to analysis the diffusion of the solvent into the gel which is directly related to the release rate.

The objective of this study is to develop an intelligent polymeric hydrogel to be used as drug carrier with self-regulated release, and targeted uni-directional release using $\gamma$-rays induced copolymerization and crosslinking. In this connection, methacrylic acid (MAAc), which is $\mathrm{pH}$-sensitive monomer bearing carboxylic acid groups which possess insensitive character to the acidic medium and allow swelling at $\mathrm{pH}$ values higher than their $\mathrm{pKa}$ value, will copolymerized with poly (2-ethyl-2-oxazoline), which is a biocompatible low cytotoxic and $\mathrm{pH}$-sensitive polymer with favorable pKa value near neutral (Wang et al., 2011 and Jabbari et al., 2018). The parameters affecting the copolymerization process such as feed solution composition and concentration and irradiation dose will be optimized. The prepared hydrogels will be characterized by investigating their swelling characteristics. The ability of the prepared hydrogels to perform as a $\mathrm{pH}$-sensitive drug carrier for colon specific drug delivery system will be evaluated using Ibuprofen as a model drug. Ibuprofen is an effective is a non-steroidal anti-inflammatory drug. Ibuprofen is a core medicine in the World Health Organization's "Essential Drugs List", which is a list of minimum medical needs for a basic health care system

\section{MATERIALS}

Methacrylic acid (MAAc) of purity $99.9 \%$ and poly(2-ethyle-2-oxazoline)(PEOz), purchased from (Aldrich, Germany), were used as received. Ibuprofen, pharmaceutical grade, is kindly provided by Dar Al-Hekma Pharmaceutical Co., Alsalt, Jordon. Citric acid, Sodium citrate, Sodium dihydrogen phosphate, and Disodium hydrogen phosphate, analytical reagents, were analytical grade, purchased from Winlab, UK. 


\section{Preparation of MAAc/PEOz, hydrogels.}

MAAc/PEOz hydrogels were obtained by $\gamma$-radiation-induced homo/ copolymerization of mixtures of different compositions in glass vials. The ${ }^{60} \mathrm{Co}$ gamma radiation source used is Nordion 2.2 at dose rate of $10 \mathrm{kGy} / \mathrm{h}$. The irradiation facilities are established by National Center for Radiation Research and Technology, Egyptian Atomic Energy Authority, Cairo, Egypt. All samples were cutted into disks, washed in excess water to remove the unreacted component then air dried at room temperature up to constant weight.

\section{Preparation of Buffer Solutions of Different pH's:}

(Citric acid/trisodium citrate) and (Sodium dihydrogen phosphate/ disodium hydrogen phosphate) were used to prepare buffer solutions ranged from 3-5 and 6-7, respectively. $\mathrm{HCl}$ was used to prepare solutions of $\mathrm{pH} 1$.

\section{Swelling Study}

Dynamic swelling experiments were performed in buffer solutions of different $\mathrm{pH}$ at $37 \pm 0.2^{\circ} \mathrm{C}$. Swollen gels removed from the swelling medium at regular intervals were dried superficially with filter paper, weighed and placed in the same bath. The measurements were continued until a constant weight was reached for each sample. The amount of solution absorbed was monitored gravimetrically. The degree of swelling (S\%) was calculated using the following:

$$
\mathrm{S} \%=\frac{\left(\mathrm{W}_{\mathrm{S}}-\mathrm{W}_{\mathrm{o}}\right)}{\mathrm{W}_{\mathrm{o}}} \times 100
$$

where $\mathrm{W}_{\mathrm{o}}$ and $\mathrm{W}_{\mathrm{s}}$ are the weights of the xerogel at time 0 and of the hydrogel swollen at different buffer solutions, respectively.

\section{Preparation of drug-loaded hydrogel.}

For the investigation of drug release behavior of MAAc/PEOz hydrogels prepared in this study, ibuprofen was used as model drug. MAAc/PEOz xerogels of different compositions were immersed in saturated aqueous solution of ibuprofen at room temperature until equilibrium and the drug loaded gels were dried at room temperature.

\section{Drug release experiments}

The drug release experiments were performed by placing the drug loaded hydrogel in $25 \mathrm{ml}$ of a $\mathrm{pH} 1$ solution for $3.5 \mathrm{~h}$ then moved to $250 \mathrm{ml}$ of a $\mathrm{pH} 7$ buffer solution. The buffer solution was stirred constantly. To follow the release of the drug, aliquots were drawn from the solution at various times and the absorption at $272 \mathrm{~nm}$ was measured using a UV spectrophotometer (Perkin Elemer, Lmbda1 UV-Vis spectrophotometer). The concentration of the drug in the external solution at any selected time $\left(M_{t}\right)$ was calculated from the corresponding calibration curve of the absorption against drug concentration. From this value, the amount of drug released at the selected time was estimated.

\section{RESULTS AND DISCUSSION}

Several Techniques were used to fabricated hydrogels include cross-linking of either linear polymers or simultaneous polymerization of monofunctional monomers and cross-linking with polyfunctional monomers. Usually, polymers containing hydroxyl, amine, amide, ether, carboxylate and sulfonate as functional groups in their side chains are used. Such polymers could be of natural, synthetic or semi-synthetic origin. There are many parameters control Radiation fabrication of polymeric hydrogels. In the following some of these parameters will be optimized.

\section{Effect of Feed solution composition, concentration and irradiation dose on gelation degree}

Figure (1) shows the dependence of conversion and gelation degrees of the prepared MAAc/PEOz copolymer hydrogel on the MAAc content in the feed solution. The figure clearly shows that that pure methacrylic, as radiation crosslinkable monomer, undergo almost $100 \%$ gelation that it is able to produce 
homo-polymer upon exposure to ionizing radiation whereas the introduction of PEOz to the feed solution leads to an obvious decrease in either conversion and gelation process. Such decrement is improved by the further increase in PEOz content in the feed solution to reach the lowest conversion/gelation degrees at 35 wt $\%$ of PEOz. Further increment in PEOz content in the feed solution improve the conversion/gelation degrees to achieve maximum values; about $100 \%$, at $65 \mathrm{wt} \%$ of PEOz. The abovementioned results can be explained by assuming that PEOz, which is, is able to form strong electrolytic complex with methacrylic acid (Su et al., 2017, Ruiz-Rubio et al., 2018). Such complex formation process would lead to the grafting of acrylic acid to the PEOz chains. Such complexation will separate the grafted acrylic acid which will minimize the possibility of the homo-polymerization and self-bridging. By increasing the PEOz content in the feed solution, the mixture viscosity increases and as a result, the possibility of homo-polymerization and self-bridging increases, which in consequently leads to the increment in the grafted chain length (Ruiz-Rubio et al., 2015). The increase in the degree of branching in the addition to the ability for complex formation would lead to the formation of highly entangled structure which is not easy to be dissolved even at boiling water.

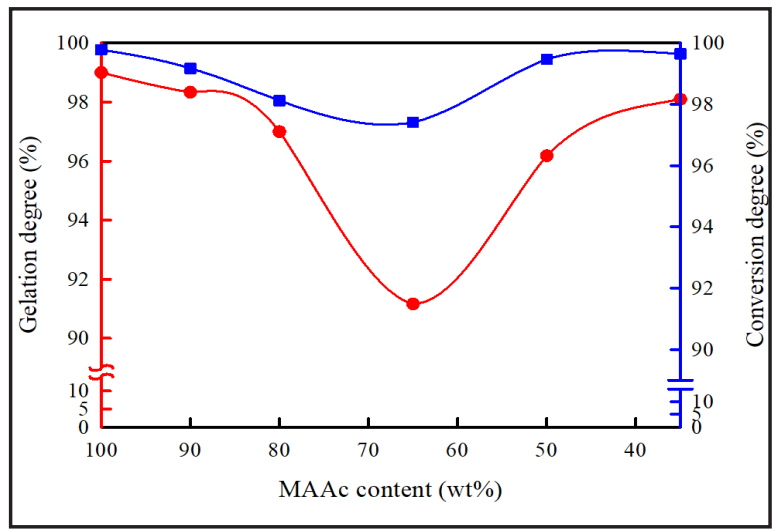

Fig. (1): Effect of the aqueous feed solution composition on the $(\bullet)$ conversion and $(\bullet)$ gelation degree of PEOz/MAAc copolymer. Irradiation dose: $15 \mathrm{kGy}$ and Feed solution concentration $15 \mathrm{wt} \%$.
On the other hand Figure (2) shows the effect of irradiation dose on the gelation degree of MAAc/ PEOz copolymer hydrogel prepared at different feed solution concentration. For all of the investigated feed solution concentrations, the data show that the increment in the adsorbed irradiation dose leads to an obvious improvement in the gelation degree. Moreover, the data show that at the same irradiation dose, the gelation degree increases as the total feed solution concentration increases. It is well known that radiation polymerization reaction is a free radical reaction (Hong et al., 2018). Increasing the irradiation dose prolong the time of exposure to the ionizing radiation i.e. increase both initiation and propagation steps of the free radical polymerization reaction which directly increases the homo/copolymerization and bridging between the formed/grafted chain resulting in perfect crosslinking structure. In the same manner, increasing the total feed solution concentration increases the number of free radicals formed per unit volume of the reaction medium which increase the probability of the recombination between the formed radical resulting in perfect crosslinking structure(Chapiro, 1995).

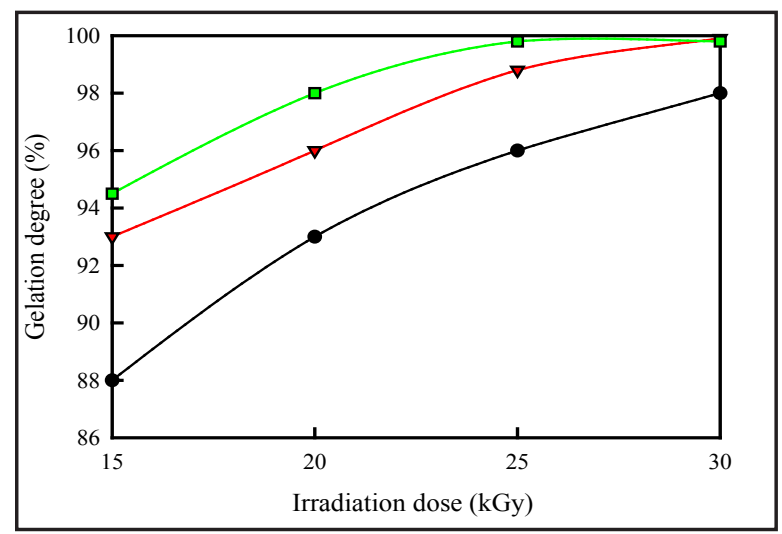

Fig. (2): Effect of the Irradiation dose on the gelation degree of PEOz/AAc copolymer at different feed solution concentration; $(\bullet) 15,(\boldsymbol{\nabla}) 20$ and $(\boldsymbol{\bullet}) 25 \mathrm{wt} \%$. Aqueous feed solution composition(MAAc/PEOz): (50:50 wt $\%$ ). 
Characterization of the prepared MAAc/PEOz copolymer hydrogel

\section{1. $\quad$ H Dependent Swelling}

The designing of good drug targeting carriers is largely dependent on the dynamics of response to the external $\mathrm{pH}$. The performance of such hydrogels as carrier for drug delivery systems can be evaluated by studying their swelling behavior in buffer solutions of different $\mathrm{pH}$ values. Figure 3 showed the $\mathrm{pH}$ dependent swelling of MAAc/PEOz hydrogel. From the figure, it can be seen that the MAAc/PEOz hydrogels possessed low swelling degree at low $\mathrm{pH}$ values; < pH 4 whereas, they possessed dramatic increases in the swelling degree at $\mathrm{pH}$ values $>\mathrm{pH}$ 4 which is a typical $\mathrm{pH}$-dependent phase transition. Such phase transition can be attributed to the dissociation state of the ionizable carboxylic groups of acrylic acid (Zhou and Chu, 1998). Below pH 4 i.e. below pKa of carboxylic acid, the carboxylic groups are completely associated forming inter- and/or intramolecular hydrogen bonding resulted in the collapse of the hydrogel (Nagy 2014). However, at $\mathrm{pH}$ values higher than $\mathrm{pH} 4$, the carboxylic groups start to dissociate forming carboxylate ions.

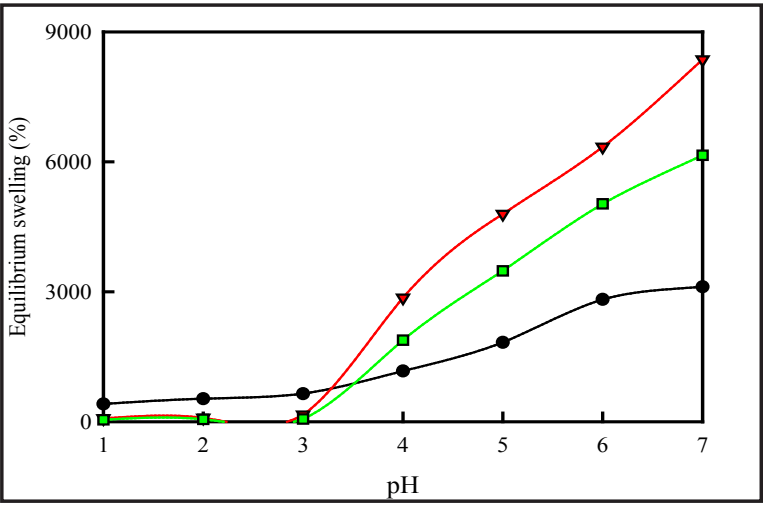

Fig. (3): pH-dependent swelling of MAAc/PEOz copolymer hydrogels of different MAAc content; $(\bullet)$ 35, (•) 50 and $(\boldsymbol{\nabla}) 90 \mathrm{wt} \%$. Feed solution concentration $20 \mathrm{wt} \%$, irradiation dose $20 \mathrm{kGy}$.
The electrostatic repulsion between the carboxylate ions which resulted in an increase in the free spaces available for swelling in addition to the hydrophilicity of such carboxylate ions would make that remarkable observed increase in the swelling degree along the phase transition (Ofner and Schott, 1985).

\section{Swelling Kinetics}

To evaluate the performance and ability of a prepared polymeric hydrogel to serve as a carrier for drug delivery systems. Analysis of the fluid transport in swellable polymers is important to understand the diffusion mechanism. Swelling kinetics of the prepared MAAc/PEOz copolymer hydrogels were studied as a function of solution $\mathrm{pH}$ values. For the determination of the swelling mechanism, diffusion exponent " $n$ " was obtained from samples swelling in buffer solutions of $\mathrm{pH} 1$ and 7. Typical dynamic swelling curves of MAAc/PEOz copolymer hydrogel containing equi MAAc / $\mathrm{PEOz}$ weight at $\mathrm{pH} 1$ and 7 are demonstrated in Figures 4 and 5. Figure 4 shows the time dependent swelling of MAAc / PEOz copolymer hydrogel at $\mathrm{pH} 1$ and 7. MAAc/PEOz hydrogel showed a gradual and a very low swelling degree at $\mathrm{pH} 1$, while the opposite occurred at $\mathrm{pH} 7$. The water uptake equilibrium for the hydrogel from pH 7 buffer solution was about $400 \mathrm{wt} \%$ which was about 4 times higher than those obtained at $\mathrm{pH} 1$ buffer solution. Such behavior could be attributed to the presence of MAAc which acted as a swelling controlling agent. At $\mathrm{pH}$ values lower than the $\mathrm{pKa}$ of AAc the swelling processes were more restrict due to the formation of hydrogen bonding which enhanced the crosslinking density of the hydrogel, thus AAc serves as pseudo crosslinker. On the other hand, at $\mathrm{pH} 7$ the formed hydrogen bonds were disconnected and the repulsion between the dissociated carboxylate groups increased the water uptake rate. 


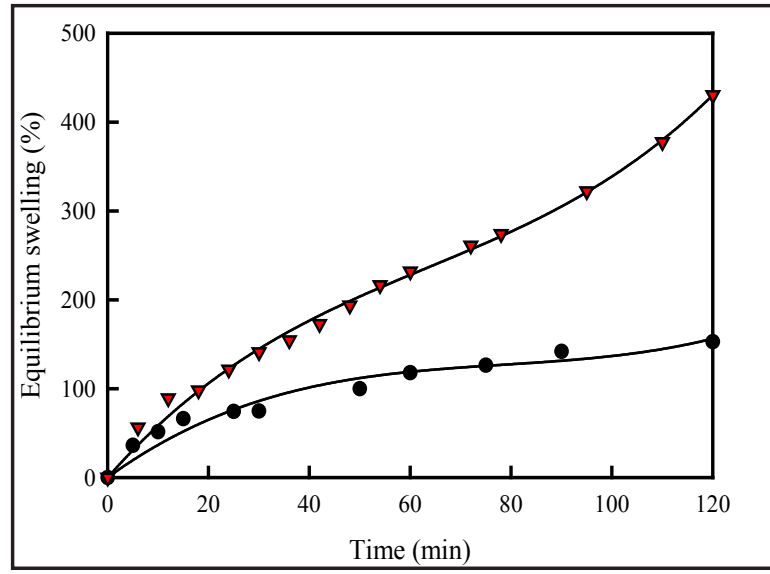

Fig. (4): Time dependent swelling of equi-weight MAAc/ $\mathrm{PEOz}$ at $(\bullet) \mathrm{pH} 1$ and $(\boldsymbol{\nabla}) \mathrm{pH} 7$.

For most polymeric hydrogels, swelling and drug release could be explained in terms of the simple diffusion for water uptake by the hydrogel which leads to the dissolution and release of the drug from the polymeric matrix. Fick's law of diffusion and useful approximation are applied to estimate the diffusion type through the polymeric matrix (Yin et al., 2010 and Wei et al., 2013). The following is Fick's law of diffusion.

where $M_{t}$ and $M_{\infty}$ are the amount of drug released or water absorbed at time and equilibrium respectively, $\mathrm{K}$ is the release or adsorption constant and $\mathrm{n}$ is the swelling or release index.

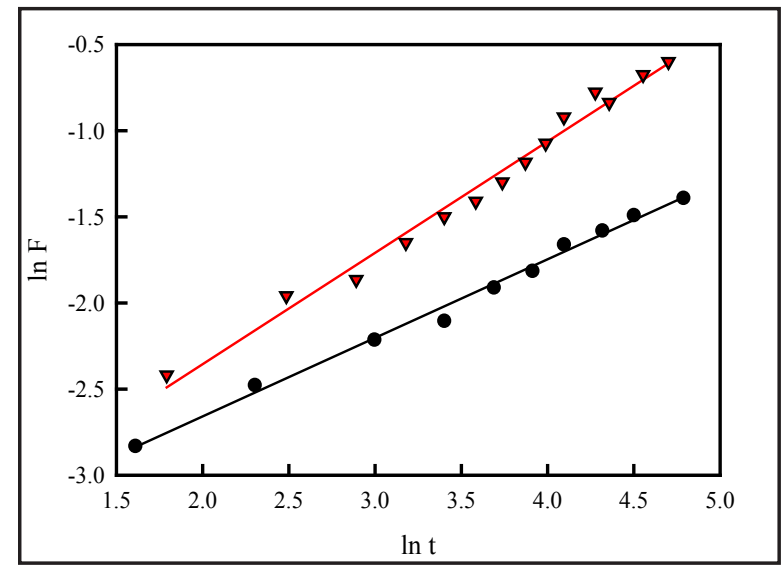

Fig. (5): Linear dependence of $\ln F$ on $\ln t$ of equi-weight MAAc/PEOz at $(\bullet)$ pH 1 and $(\boldsymbol{\nabla})$ pH 7.

The values of $\mathrm{K}$ and $\mathrm{n}$ are found by fitting the data to the above expression. For non-swollable polymeric systems, $\mathrm{n}$ was found to be around 0.5 implying that the diffusion is the controlling mechanism for water absorption and drug release which is simply called Fickian type of diffusion. However, for $0.5<\mathrm{n}<1.0$ an anomalous diffusion behavior is followed which is a time dependent mechanism called non-Fickian type of diffusion. Finally for $n=$ 1 drug release or water adsorption shows a zero order profile (Frisch, 1965; Rehage et al., 1970 and Fu and Kao, 2010).

Table (1): Calculated values for swelling indices of MAAc/PEOz copolymer hydrogel of different compositions in buffer solutions of $\mathrm{pH} 1$ and.

\begin{tabular}{|c|c|c|c|c|c|c|}
\hline \multirow{2}{*}{$\begin{array}{c}\text { MAAc } \\
\text { content } \\
(\mathbf{w t} \%)\end{array}$} & \multicolumn{5}{|c|}{ pH 1 } & $\mathbf{n}$. \\
\cline { 2 - 7 } & $\mathbf{n}$ & $\mathbf{k}$ & $\mathbf{r}^{\mathbf{2}}$ & $\mathbf{n}$ & $\mathbf{k}$ & $\mathbf{r}^{\mathbf{2}}$ \\
\hline 90 & 0.45 & -3.42 & 0.99 & 0.81 & 2.94 & 0.99 \\
\hline 50 & 0.49 & -3.84 & 0.99 & 0.75 & 3.70 & 0.99 \\
\hline 35 & 0.51 & -4.35 & 0.99 & 0.54 & 3.89 & 0.96 \\
\hline
\end{tabular}


Swelling indices were calculated to investigate the effect of both copolymer composition and solution $\mathrm{pH}$ values on the diffusion type. Figure 5 shows the linear dependence between $\ln \mathrm{F}$ and $\ln$ $\mathrm{t}$ for MAAc/PEOz copolymer hydrogel. Table (1) provides calculated values for swelling indices of MAAc/PEOz of different compositions at buffer solutions of $\mathrm{pH} 1$ and 7 from curves similar to Figure (4 and 5). The table shows that all the samples possessed Fickian type of diffusion at $\mathrm{pH} 1$ whereas they underwent non-Fickian type at $\mathrm{pH}$ 7. The abovementioned data show that the prepared MAAc/PEOz hydrogel is a good

Candidate as a carrier for drug delivery system. The abovementioned data suggest the prepared MAAc/PEOz hydrogel as a good candidate to serve as a carrier for the drug delivery system

Potential applicability of MAAc/PEOz copolymer hydrogel as pH-sensitive Drug carrier

Oral administration of the drug loaded gel reach the stomach $(\mathrm{pH} 1-2.5)$ through gullet, then pass into the terminal ileum $(\mathrm{pH} 7.5 \pm 0.4)$ from the proximal small intestine $(\mathrm{pH} 6 \pm 0.5)$ and the colon $(\mathrm{pH}$ 6.5), where the release of drugs usually involves the simultaneous absorption of gastrointestinal juice resulting largely from $\mathrm{pH}$ and desorption of the drug via swelling-control diffusion mechanism(Fallingborg, 1999). In this respect, to evaluate the $\mathrm{MAAc} / \mathrm{PEOz}$ for the possible use in colon drug delivery system, Ibuprofen was loaded to the MAAc/PEOz hydrogels. The drug loaded gel were immersed at buffer solution of $\mathrm{pH} 1$ for 210 minutes (mean gastric residence time) followed by immersing in buffer solution of $\mathrm{pH} 7$ for 16 hours (far in excess for small intestine time), and the amount of drug released was determined. Figures (6 and 7) show the $\mathrm{pH}$ sensitive release profiles of Ibuprofen from $\mathrm{MAAc} / \mathrm{PEOz}$ hydrogel of different AAc contents by immersing Them in buffer solution of $\mathrm{pH}$ 1, Figure (6), and then in buffer solution of $\mathrm{pH} 7$, Figure (7).

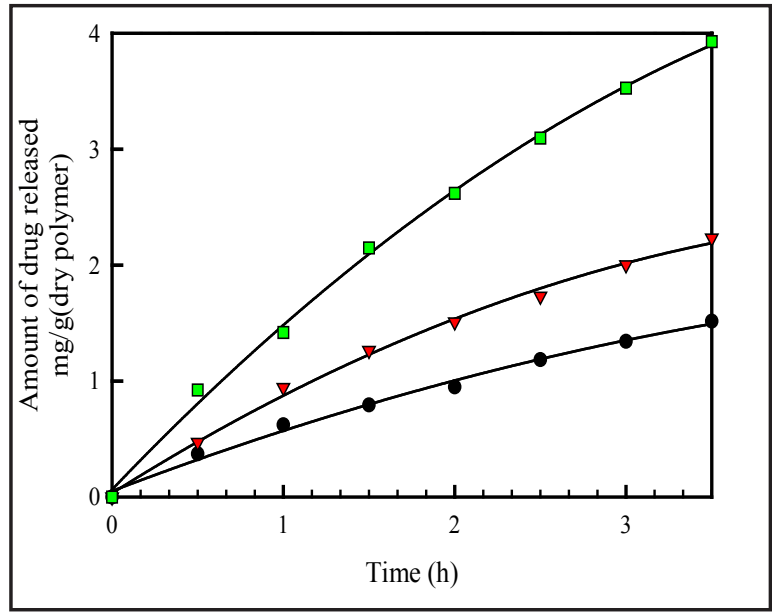

Fig. (6): Drug release profile of Ibuprofen as model drug from MAAc/PEOz copolymer hydrogel of different MAAc content; ( $\bullet$ ) 35, ( $\boldsymbol{\nabla})$ 50, and $(\bullet) 90 \mathrm{wt} \%$ at buffer solution of $\mathrm{pH}$.

The data shows that there is no drug release in the stomach media i.e. at $\mathrm{pH}$ 1. By changing $\mathrm{pH}$ from 1 to 7 , drastic increase in drug release was observed. Continuous release of Ibuprofen was noticed during the immersion time to reach its maximum after eighteen hours thereafter tends to level off. Also, it was observed that the amount of drug released from $\mathrm{P}$ (MAAc) rich gel is higher than that of gel containing less amount of $\mathrm{P}(\mathrm{MAAc})$. The rate of release increases as the swelling increase.

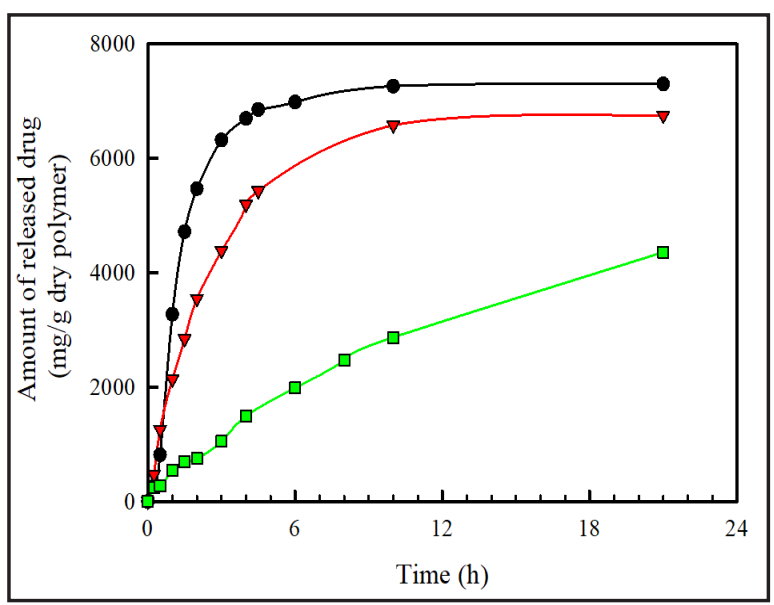

Fig. (7): Drug release profile of Ibuprofen as model drug from MAAc/PEOz copolymer hydrogel of different MAAc

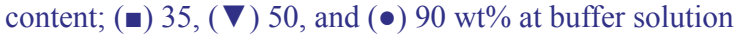
of $\mathrm{pH} 7$. 


\section{ACKNOWLEDGEMENT}

Author would like to express his thanks and gratitude for polymer chemistry department, National Center for Radiation Research and Technology, Egyptian atomic Energy Authority for the generous hospitality and the facility provided during the period of hosting him in Cairo, Egypt.

\section{REFERENCES}

- Aqil, F.; Munagala, R.; Jeyabalan, J. and Vadhanam, M.V. (2013): Bioavailability of phytochemicals and its enhancement by drug delivery systems. Cancer Lett., 334(1): 133.

- Chapiro, A. (1995): General consideration of the radiation chemistry of polymers. Nucl. Instrum. Methods Phys. Res. B, 105(1-2): 5.

- $\quad$ Davoodi, P.; Lee, L.Y.; Xu, Q.; Sunil, V.; Sun, Y.; Soh, S. and Wang, C.H. (2018): Drug delivery systems for programmed and on-demand release. $A d v$ Drug Deliv. Rev., 132: 104

- Dragan, E.S. (2014): Design and applications of interpenetrating polymer network hydrogels. A review. Chem. Eng. J., 243: 572.

- Fallingborg, J. (1999): Intraluminal pH of the human gastrointestinal tract. Dan. Med. Bull., 46(3): 183.

- Florence, A.T. (2018): Nanotechnologies for site specific drug delivery: changing the narrative. Int. J. Pharm., 551(1-2): 1.

- Frisch, H.L. (1965): Mechanisms for fickian diffusion of penetrants in polymers. J. Polym. Sci. B, 3(1): 13.

- Fu, X.; Hosta-Rigau, L.; Chandrawati, R. and Cui, J. (2018): Multi-Stimuli-Responsive Polymer Particles, Films, and Hydrogels for Drug Delivery. Chem., 4(9): 2084.

- Fu, Y. and Kao, W.J. (2010): Drug Release Kinetics and Transport Mechanisms of Non-degradable and Degradable Polymeric Delivery Systems. Expert Opin. Drug Deliv., 7(4): 429.
- Hong, T.T.; Okabe, H.; Hidaka, Y. and Hara, K. (2018): Radiation synthesis and characterization of super-absorbing hydrogel from natural polymers and vinyl monomer. Environ. Pollut., 242: 1458.

- Jabbari, S.; Ghamkhari, A.; Javadzadeh, Y.; Salehi, R. and Davaran, S. (2018): Doxorubicin and chrysin combination chemotherapy with novel $\mathrm{pH}$-responsive poly [(lactide-co-glycolic acid)-blockmethacrylic acid] nanoparticle. J. Drug Deliv. Sci. Tec., 46: 129.

- Kanamala, M.; Palmer, B.D.; Wilson, W.R. and Wu, Z. (2018): Characterization of a smart pH-cleavable PEG polymer towards the development of dual pH-sensitive liposomes. Int. J. Pharm., 548(1): 288.

- Kohrs, N.J.; Liyanage, T.; Venkatesan, N.; Najarzadeh, A. and Puleo, D.A. (2018): Drug Delivery Systems and Controlled Release. Reference Module in Biomedical Sciences, Elsevier.

- $\quad$ Li, X.X.; Chen, J.; Shen, J.M.; Zhuang, R.; Zhang, S.Q.; Zhu, Z.Y. and Ma, J.B. (2018): pH-Sensitive nanoparticles as smart carriers for selective intracellular drug delivery to tumor. Int. J. Pharm., 545(1): 274.

- Mahinroosta, M.; Jomeh Farsangi, Z.; Allahverdi, A. and Shakoori, Z. (2018): Hydrogels as intelligent materials: A brief review of synthesis, properties and applications. Mater. Today Chem., 8: 42.

- Nagy, P. I. (2014): Competing Intramolecular vs. Intermolecular Hydrogen Bonds in Solution. Int. J. Mol. Sci., 15(11): 19562.

- Ofner, C. M. and Schott, H. (1985): Shifts in the apparent ionization constant of the carboxylic acid groups of gelatin. J. Pharm Sci., 74(12): 1317.

- $\quad$ Rehage, G.; Ernst, O. and Fuhrmann, J. (1970): Fickian and non-Fickian diffusion in high polymer systems. Faraday Discuss., 49(0): 208.

- Ruiz-Rubio, L.; Alonso, M.; Pérez-Álvarez, L.; Alonso, R.; Vilas, J. and Khutoryanskiy, V. (2018): Formulation of Carbopol@/Poly(2-ethyl-2-oxazoline) s Mucoadhesive Tablets for Buccal Delivery of Hydrocortisone. Polymers, 10(2): 175. 
- Ruiz-Rubio, L.; Álvarez, V.; Lizundia, E.; Vilas, J.L.; Rodríguez, M. and León, L.M. (2015): Influence of $\alpha$-methyl substitutions on interpolymer complexes formation between poly(meth)acrylic acids and poly(N-isopropyl(meth)acrylamide)s. Colloid Polym. Sci., 293(5): 1447.

- Sakr, F.M. (1999): A programmable drug delivery system for oral administration. Int. J. Pharm., 184(1): 131 .

- Sarpietro, M.G. and Castelli, F. (2013): Drug delivery systems: drug nanocarriers. Drug-Biomembrane Interaction Studies. R. Pignatello, Woodhead Publishing: 335 .

- Sher, P.; Ingavle, G.; Ponrathnam; S. and Pawar, A.P. (2007): Low density porous carrier based conceptual drug delivery system. Microporous and Mesoporous Mater., 102(1): 290.

- $\quad$ Streisand, J. B. and Stanley, T.H. (1995): Newer drug delivery systems. Curr. Anaesth. Crit. Care, 6(2): 113.

- Su, C.; Sun, J.; Zhang, X.; Shen, D. and Yang, S. (2017): Hydrogen-Bonded Polymer Complex Thin Film of Poly(2-oxazoline) and Poly(acrylic acid). Polymers, 9(8): 363.

- Szewczyk, A.; Prokopowicz, M.; Sawicki, W.; Majda, D. and Walker, G. (2019): Aminopropyl-functionalized mesoporous silica SBA-15 as drug carrier for cefazolin: adsorption profiles, release studies, and mineralization potential. Microporous and Mesoporous Mater, 274: 113.
- Ullah, F.; Othman, M.B.H.; Javed, F.; Ahmad, Z. and Akil, H.M. (2015): Classification, processing and application of hydrogels: A review. Mat. Sci. Eng. C, 57: 414.

- Wang, X.; Li, X.; Li, Y.; Zhou, Y.; Fan, C.; Li, W.; Ma, S.; Fan, Y.; Huang, Y.; Li, N. and Liu, Y. (2011): Synthesis, characterization and biocompatibility of poly(2-ethyl-2-oxazoline)-poly(d,1-lactide)poly(2-ethyl-2-oxazoline) hydrogels. Acta Biomater., 7(12): 4149.

- Wang, Y.; Chen, B.Z.; Liu, Y.J.; Wu, Z.M. and Guo, X.D. (2017): Application of mesoscale simulation to explore the aggregate morphology of $\mathrm{pH}$ sensitive nanoparticles used as the oral drug delivery carriers under different conditions. Colloids Surf. B, 151: 280 .

- Wei, Q.B.; Luo, Y.L.; Fu, F.; Zhang, Y.Q. and Ma, R.X. (2013): Synthesis, characterization, and swelling kinetics of $\mathrm{pH}$-responsive and temperatureresponsive carboxymethyl chitosan/polyacrylamide hydrogels. J. Appl. Polym. Sci., 129(2): 806.

- $\quad$ Yin, Y.; Lv, X.; Tu, H.; Xu, S. and Zheng, H. (2010): Preparation and swelling kinetics of $\mathrm{pH}$-sensitive photocrosslinked hydrogel based on carboxymethyl chitosan. J. Polym. Res. 17(4): 471.

- Zhou, S. and Chu, B. (1998): Synthesis and Volume Phase Transition of Poly (methacrylic acid-co-Nisopropylacrylamide) Microgel Particles in Water. $J$. Phys. Chem. B, 102(8): 1364. 
